

\section{Chest physical therapy in surgery: a theoretical model about who to treat}

\section{Educational aims}

I To highlight high- and low-risk factors for PPC.

I To present overview data showing how the respiratory system is affected by different surgical interventions.

I To present a model to determine which treatment is most suitable for each individual patient.

\section{Summary}

Chest physiotherapy is common for patients undergoing different types of surgery in order to avoid PPC. However, do all patients require treatment, is it possible to predict those patients at risk and is it possible to select the optimal treatment for each patient? This paper aims to address these questions and to propose a model that can be utilised for determining how much physical therapy is needed.

Since the early 1900s, physiotherapists have treated surgical patients to prevent post-operative pulmonary complications (PPC) [1]. Chest physiotherapy originated in the UK and, in 1915, one of the first articles was published describing breathing exercises to restore respiration following damage to the pleura, lung and diaphragm [2]. The techniques included deep breathing exercises in the recumbent position and, if necessary, with the body bent laterally away from the injured side. The treatment required support from an "operator" who, for example, placed his or her hands on the area of the thorax where the collapse was. The earliest time at which the exercises were commenced was 5 weeks from the day of the injury [2].

These techniques, albeit modified, have been used since the conception of chest physiotherapy, but, during development, new treatments and regimens have been created. Different manual treatment methods, including percussion, clapping, vibrations and shaking, have been, and in some parts of the world still are, common $[3,4]$. Position changing for bronchial drainage to decrease the risk of atelectasis is another method employed for the prevention of pulmonary complications [3-5].

Over the last few decades, treatments have changed, becoming more active, whereby the patients are encouraged to carry out a larger part of the training by themselves $[6,7]$. The use of deep breathing exercises has developed with the introduction of different breathing devices, including incentive spirometry and positive expiratory pressure (PEP), to facilitate breathing post-operatively and to accelerate the
M. Fagevik Olsén

Dept of Physical Therapy Sahlgrenska University Hospital S-413 45 Gothenburg Sweden

Fax: 46313424341

E-mail: monika.fagevikolsen@vgregion.se 
Figure key

$\longrightarrow$ Hysterectomy $\mathrm{n}=20$

$\Longrightarrow$ Bowel $n=82$

—- Fundoplication $n=35$

- Upper gastrointestinal $\mathrm{n}=32$

- Gastroplasty $n=47$ Heart $n=55$

- Thoracoabdominal $n=35$

—- Laparoscopic hysterectomy $n=20$

—- Laparoscopic fundoplication $n=20$

_- Laparoscopic vertical banded gastroplasty $n=20$

-_ Laparoscopic gastric bypass $n=20$

A key for figures 1-3 showing all lines with the associated operation and number of patients studied.

\section{Figure 1}

$\mathrm{Sa}, \mathrm{O}_{2}$ before and after different open procedures.

\section{Figure 2}

Changes in FVC after different open and laparoscopic procedures (pre-operative value is $100 \%$ ). return to pre-operative respiratory status [6-9]. Another development in the care of post-operative patients is the change of routines concerning mobilisation. The previous long-term immobility after surgery has been replaced by early mobilisation, which is now a main aim of treatment $[2$, 10].

To avoid PPC, patients undergoing major surgery often receive pre-operative information from a physical therapist, including breathing exercises with or without breathing devices, techniques for facilitating mucus flow (e.g. forced expiratory technique) and the importance of early mobilisation. Post-operatively, treatment continues according to the patient's respiratory status.

However, do all patients require treatment, and is it possible to predict those patients at risk and thereby select the optimal treatment for each patient? The aims of this article are to point out high- and low-risk factors for PPC, present overview data showing how the respiratory system is affected by different surgical interventions and to present a model to determine which treatment is most suitable for each individual patient.
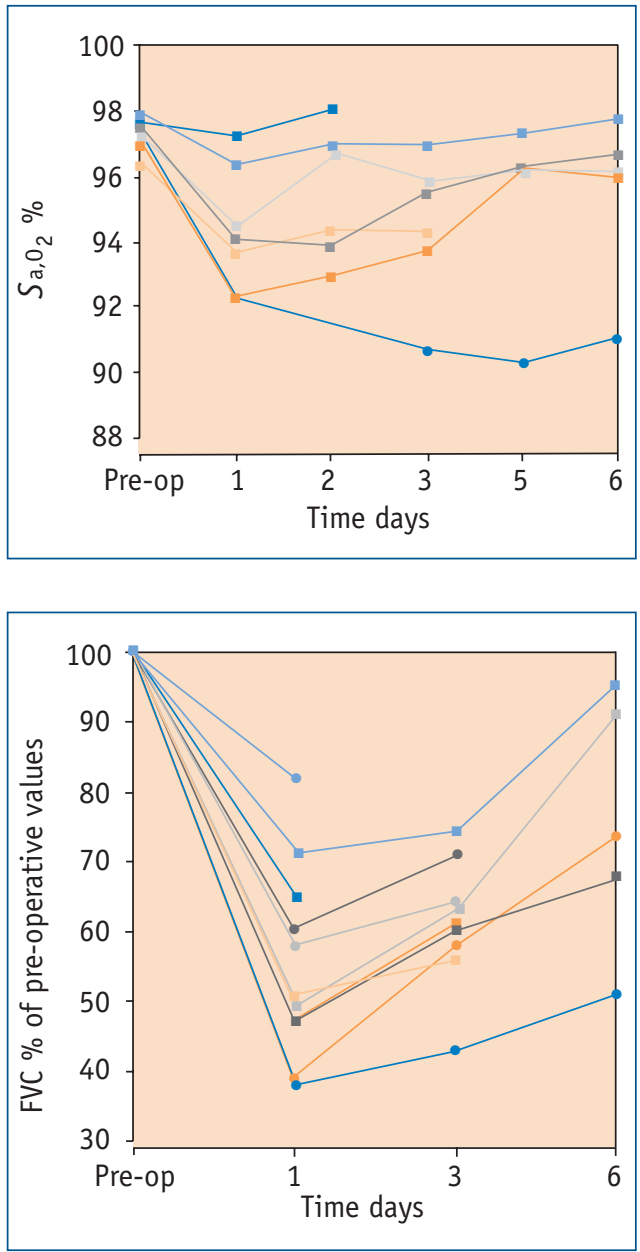

\section{Risk factors}

Patients undergoing surgery are at varying risks of developing PPC. Some of the major contributing factors are listed as follows.

\section{Advanced age}

The pulmonary system changes during adult lifetime. For instance, closing volume and functional residual capacity (FRC) increase linearly with age and closing volume exceeds expiratory reserve volume, giving rise to smaller airway collapses in persons aged $\geq 44$ years in the supine position and at $\sim 65$ years in the sitting position $[11,12]$. Many trials confirm that the older the patient and the greater the surgical procedure, the greater the morbidity and mortality after major surgery. The high-risk age level is reported to be from 55 years [13].

\section{Smoking}

Smoking causes major changes in the pulmonary system, e.g. reduction in lung capacity, increased shunt, production of mucus and impairment of the normal mechanics of mucus clearance, such as ciliary activity, leading to productive coughing $[4,14]$. There is a connection between frequent smoking and increased risk of PPC [12], and a level of $>20$ pack-years ( 1 pack-year $=1$ package a day for 1 year) has been suggested to imply a higher risk of PPC [15].

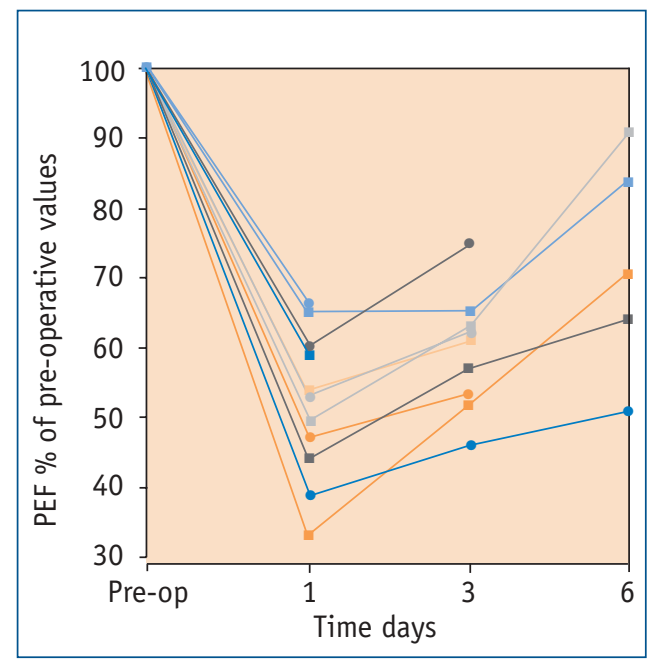

Figure 3

Changes in forced PEF after different open and laparoscopic procedures (pre-operative value is $100 \%)$. 


\section{Obesity}

Obesity is defined as a body mass index (BMI) $>30$, and very severe obesity as a BMI $>40$ [16]. Obesity impairs lung volumes, decreases respiratory compliance and increases respiratory resistance [17-19]. Obese subjects have been reported to have up to twice the normal total respiratory work values [19]. In the post-operative period, the decreased ventilation in overweight persons implies a higher risk of development of PPC compared to persons of a normal weight [13, $20,21]$.

\section{Obstructive pulmonary diseases}

Obstructive pulmonary disorders affect the respiratory system by causing inflammation in the airways, mucus hypersecretion and bronchospasm [4]. The airway obstruction includes both reversible factors, such as inflammation, bronchospasm or mucus plugging, and irreversible factors, such as fibrotic airway walls or damaged alveoli [4]. Patients suffering from active obstructive diseases are, therefore, at a high risk of developing PPC after major surgery [13].

\section{Operation site}

Abdominal surgery is associated with a higher risk of PPC than non-abdominal surgery, e.g. orthopaedic surgery, and upper abdominal surgery implies a higher risk than lower $[13,21,22]$. Upper abdominal surgery induces a marked diaphragmatic dysfunction lasting $\sim 1$ week postoperatively and is not affected by pain relief. The cause of the dysfunction has been suggested to be the main cause of the post-operative restrictive pulmonary pattern $[23,24]$.

\section{Duration of anaesthesia/surgical procedure}

The longer the anaesthesia lasts, the higher the morbidity and mortality rate $[13,22]$. An important factor is that more complicated surgery is associated with a longer duration of anaesthesia [22]. WIGHTMAN [25] discusses this in a trial where the incidence of PPC was higher in patients whose operation lasted $>30$ minutes, but, after further analysis, the group had a higher incidence of PPC independent of the duration of the operation. The result was assumed to be related to the type of operation performed rather than the length of anaesthesia.

\section{Post-operative immobilisation}

Surgery is, in mostcases, followed by a post-operative phase of immobilisation and decreased activity. Immobilisation leads to decreased lung volumes, which increases the risk of developing PPC; the less erect the body is, the lower the gas level in the lungs [5]. However, during slumped sitting, lung values are equivalent to those in the supine position [26]. Mobilisation is also an important treatment in evacuating sputum, which accumulates during the period of impaired ciliary activity [4].

\section{Pain}

It is well known that post-operative pain limits the ability to take deep breaths and to be active; however, even after adequate pain relief, the impairment of pulmonary function is still considerable, leading to the hypothesis that there is a non-analgesic-dependent dysfunction of inspiratory and expiratory muscles after upper abdominal surgery $[27,28]$. Some analgesics, such as morphine, also cause a dose-related ventilatory depression [27].

\section{Respiratory changes and risk of PPC}

How extensive are the changes in lung function, and how high is the risk of developing PPC when consecutive series of patients undergoing abdominal and/or thoracic surgery are followed after surgery?

Over a period of 13 years, a series of trials including different types of surgery have been performed at Sahlgrenska University Hospital (Gothenburg, Sweden). Respiratory function and risk of PPC were initially evaluated after upper abdominal surgery: gastroplasty; fundoplication; bowel procedures; and a miscellaneous group,
Figure 4

Risk of PPC after changes in FVC after different open and laparoscopic procedures.

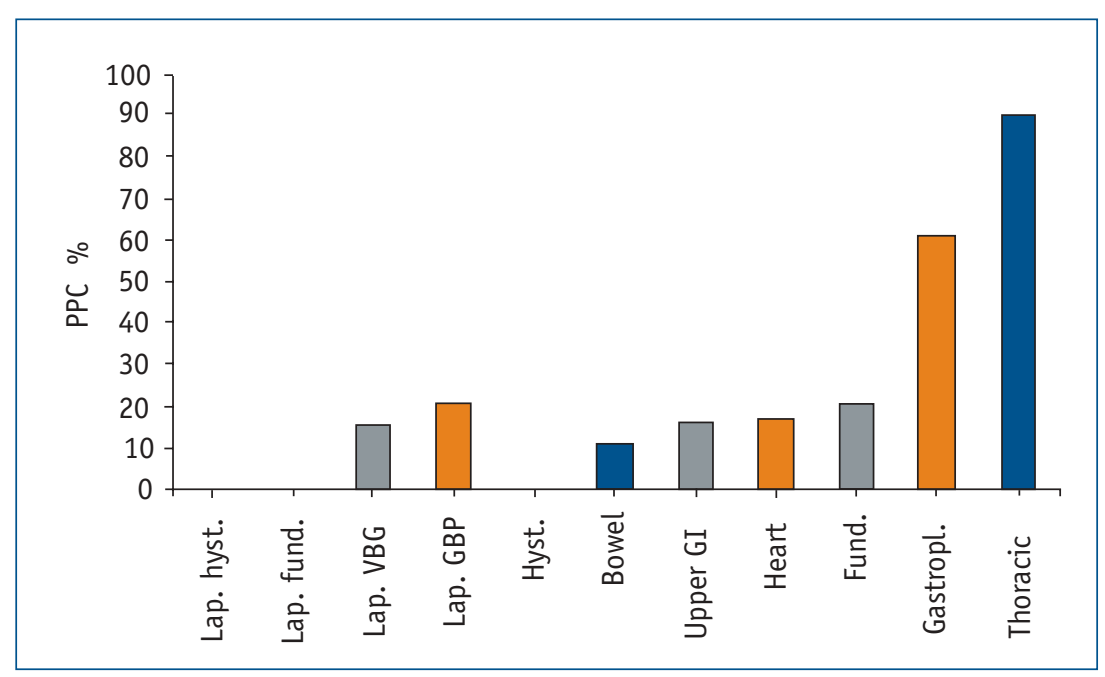


including gastrectomies, whipples and different kinds of pancreas surgery [20]. Subsequently, studies concerning open and laparoscopic hysterectomy [29], laparoscopic fundoplication [30], laparoscopic gastroplasty, as vertical banded gastroplasty [30] and gastric bypass [31], open heart surgery and thoracoabdominal oesophagus surgery [32] have been performed.

All values presented in this review are from a non-treated control groups, except for the figures from patients undergoing heart surgery and thoracoabdominal surgery, who had performed breathing exercises (three sessions of 10 breaths) with a PEP mask (Astra Tech AB, Mölndal, Sweden) every 2 nd hour versus continuous positive airway pressure (CPAP) for 30 minutes every 2nd hour during the daytime. In all series, oxygen saturation $\left(\mathrm{Sa}_{1} \mathrm{O}_{2}\right)$, forced vital capacity (FVC) and the ability to perform a maximal expiration, such as peak expiratory flow (PEF), were measured in a standardised manner in the sitting position [33].

The criteria for pulmonary complication were, for all trials, as follows: $\mathrm{Sa}_{1} \mathrm{O}_{2}<92 \%$ or pneumonia, as the findings of chest radiography verified pneumonary infiltrates in combination with fever or pathological lung auscultation. The effects of anaesthesia and surgery on measured variables are presented in figures $1-4$. The procedure's influence was highest in patients undergoing thoracoabdominal surgery or open obesity surgery, but laparoscopic surgery was also found to impair ventilation considerably post-operatively. The risk of PPC also increased with the extent of surgery: the greatest risk found with major open procedures and thoracoabdominal surgery.

\section{Which patients require treatment to avoid PPC}

As stated previously, older patients, smokers, and patients suffering from obesity or obstructive lung disease are at a greater risk of developing PPC when compared to patients without these factors. However, the type of surgery performed must also be taken into consideration when prescribing physical therapy after surgery.

A theoretical model for determining the amount of physical therapy has, therefore, been created and is presented in figure 5 . The physical therapy included in each quadrant is dependent on where the model is applied, and this is described by the following examples.

\section{A gynaecological ward}

Patients in quadrant 1 (undergoing, for instance, a laparoscopic hysterectomy) do not need any prophylactic physical therapy at all $[29,34]$ and those belonging in quadrant 2 (open hysterectomy) or 3 may be given some information pre-operatively and a check-up post-operatively $[29,34]$. Patients in quadrant 4 (for instance, a 50-year-old obese female who has smoked for 30 years and is undergoing a major resection because of a malignancy) need pre-operative information, including the importance of positioning in bed and early mobilisation and instructions on how to perform deep breathing exercises and how to evacuate sputum. They may also require a treatment post-operatively.

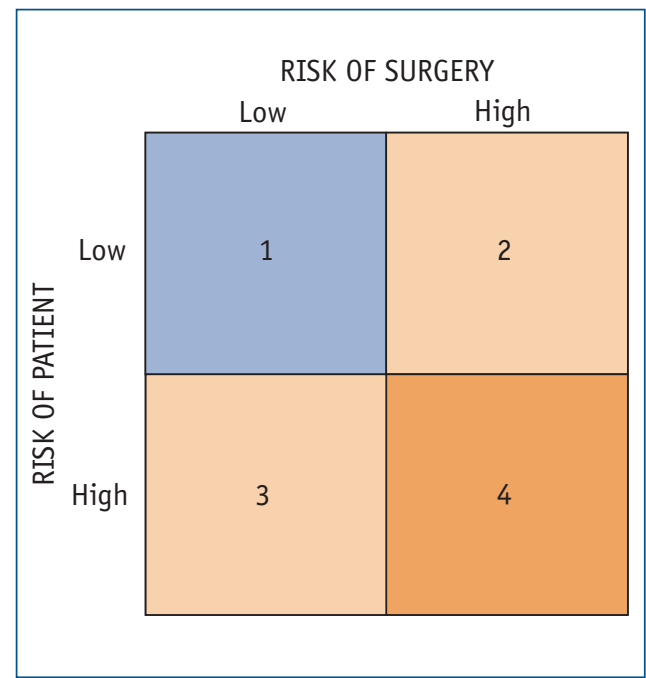

Figure 5

A theoretical model for determining the amount of physical therapy based on the patient's pre-operative status (high versus low risk) and the surgery the person will undergo (high versus low risk).

\section{Upper gastrointestinal ward}

Upper abdominal surgery may be divided into low-risk surgery (laparoscopic procedures or minor open operations, such as cholecystectomy and high-risk surgery) and all major operations. Patients in quadrant 1 do not require any prophylactic intervention at all $[30,31]$, whereas patients in quadrants 2 or 3 need pre-operative information as stated previously and post-operative treatment, and patients in field 4 require effective treatment, including more intensive breathing exercises like PEP and intensive treatments post-operatively to avoid PPC [35].

In some cases, the model may be adjusted to include other types of patient or surgery risk categories. The patients may, for instance, be 
divided into three categories: low, moderate or high risk. The model can, therefore, be adapted to suit each specific unit. Due to varying treatment traditions used globally, it is important that the unit applying the model is in agreement regarding suitable physical therapy treatments for the different patient categories. The treatment is regarded as more superior if it is based on scientific evidence. However, clinical experience remains very important, as further research is still required in order to determine which treatment is most suitable for the individual patient. The model may be a useful tool in the challenging decision of prescribing optimal treatment for each patient in order to avoid pulmonary complications after surgery.

\section{Educational questions}

1. What is the best treatment to avoid PPC?
a) Deep breathing exercises.
b) Inhalations.
c) Mobilisation.

2. Why is there a higher risk of developing PPC after upper abdominal surgery than lower?
a) The operations are more extensive in the upper abdomen.
b) The operation is performed closer to the diaphragm.

3. Smoking increases the risk of PPC. Where is the level for when smoking affects the risk of developing PPC?
a) After $>20$ pack-years.
b) Immediately after starting smoking.
c) After 40 pack-years.

4. Why is a more erect posture preferable for the lungs after surgery?
a) Posture does not affect lungs.
b) It increases perfusion.
c) The more erect the body is, the higher the gas level in the lungs.

\section{Suggested further reading}

Ricksten S-E, Bengtsson A, Söderberg $C$, et al. Effects of periodic positive airway pressure by mask on postoperative pulmonary function. Chest 1986; 89: 774-781.

One of the best articles concerning effects of chest physical therapy after abdominal surgery. The aim of the study was to analyse the prophylactic effects of CPAP and PEP by mask on post-operative radiological complications and post-operative pulmonary function after major abdominal surgery. The results showed that both systems are effective in preventing complications.

Dean E. Oxygen transport: a physiologically-based conceptual framework for the practice of cardiopulmonary physiotherapy. Physiotherapy 1994; 80: 347-355.

A conceptual framework about cardiopulmonary physiotherapy which is important to read for all of those working with cardiopulmonary patients. Includes both theory and practice, and how to measure effects of chest physical therapy.

Fagevik Olsén M. Chest physiotherapy in open and laparoscopic abdominal surgery. Phys Ther Rev 2000; 5: 125-130.

A review article about chest physical therapy after abdominal surgery. Forty-four clinical articles were found and reviewed. The conclusion was that there are beneficial effects of breathing exercises in preventing PPC, especially in high-risk patients. 


\section{Suggested answers}

1.c

2. b

3. a

4. $\mathrm{c}$

\section{References}

1. Kigin CM. Chest physiotherapy for the postoperative or traumaric injury patient. Physical Therapy 1981; 61: 1724-1736.

2. MacMahon C. Breathing and physical exercises for the use in cases of wounds in the pleura, lung and diaphragm. Lancet 1915; 2: 769-770.

3. Sutton PP, Lopez-Vidriero MT, Pavia D, et al. Assessment of percussion, vibratory-shaking and breathing exercises in chest physiotherapy. Eur J Respir Dis 1985; 66: 147-152.

4. Hough A. Physiotherapy in respiratory care. Cheltenham, Stanley Thornes Ltd, 1997.

5. Lumb AB, Nunn JF. Respiratory function and ribcage contribution to ventilation in body positions commonly used during anesthesia. Anesth Analg 1991; 73: 422-426.

6. Christensen EF, Schultz P, Jensen OV, et al. Postoperative pulmonary complications and lung function in high-risk patients: a comparison of three physiotherapy regimens after upper abdominal surgery in general anaesthesia. Acta Anaesthesiol Scand 1991; 35: 97-104.

7. Ricksten S-E, Bengtsson A, Söderberg $C$, et al. Effects of periodic positive airway pressure by mask on postoperative pulmonary function. Chest 1986; 89: 774-781.

8. Colgran, Mahoney PD, Fanning GL. Resistance breathing (blow bottle) and sustained hyperinflation in the treatment of atelectasis. Anesthesiology 1970; 32: 543-550.

9. Celli BR. Perioperative respiratory care of the patient undergoing upper abdominal surgery. Clin Chest Med 1993; 14: 253-261.

10. Dean E. Oxygen transport: a physiologically-based conceptual framework for the practice of cardiopulmonary physiotherapy. Physiotherapy 1994; 80: 347-355.

11. Greifenstein FE, King RM, Latch SS, Comroe JR. Pulmonary function studies in healthy men and women 50 years and older. J Appl Physiol 1952; 4: 641-648.

12. Leblanc $P$, Ruff F, Milic-Emili J. Effects of age and body position on "airway closure" in man. J Appl Physiol 1970; $28:$ 448-451.

13. Brooks-Brunn JA. State of science, postoperative atelectasis and pneumonia. Heart Lung 1995; 24: 94-115.

14. Nunn JF. Nunn's applied respiratory physiology. Oxford, Butterworth-Heinemann Ltd, 1993.

15. Warner MA, Divertie MB, Tinker JH. Preoperative cessation of smoking and pulmonary complications in coronary artery bypass patients. Anaesthesiology 1984; 60: 380-383.

16. WHO 1995 Physical status: the use and interpretation of anthropometry. Report of WHO expert committee. Series 854. Geneva, WHO, 1995.

17. Chen Y, Horne SL, Dosman JA. Body weight and weight gain related to pulmonary function decline in adults: a six year follow up. Thorax 1993; 48: 375-380.

18. Ray CS, Sue DY, Bray G, et al. Effects of obesity on respiratory function. Am Rev Respir Dis 1983; 128: $501-506$.

19. Sharp JT, Henry JP, Sweany SK, et al. The total work of breathing in normal and obese men. J Clin Invest 1964; 43: 728-739.

20. Fagevik Olsén M, Hahn I, Nordgren S, et al. Randomized controlled trial of the prophylactic chest physotherapy in major abdominal surgery. Br J Surg 1997; 84: 1535-1538.

21. Hall JC, Tarala RA, Hall JL, Mander J. A multivariate analysis of the risk of pulmonary complications after laparotomy. Chest 1991; 99: 923-927.

22. Pedersen $T$, Eliasen $K$, Henriksen $E$. A prospective study of mortality associated with anaesthesia and surgery: risk indicators of mortality in hospital. Acta Anaesthesiol Scand 1990; 34: 176-182.

23. Dureuil B, Cantineau J, Desmonts JM. Effects of upper or lower abdominal surgery on diaphragmatic function. Br J Anaesth 1987; 59: 1230-1235.

24. Simonneau G, Vivien A, Sartene $R$, et al. Diaphragm dysfunction induced by upper abdominal surgery. Role of postoperative pain. Am Rev Respir Dis 1983; 128: 899-903.

25. Wightman JA. A prospective survey of the incidence of postoperative pulmonary complications. Br J Surg 1968; 55: 85-91.

26. Jenkins SC, Soutar SA, Moxham J. The effects of posture on lung volumes in normal subjects and in patients pre- and post-coronary artery surgery. Physiotherapy 1988; 74: 492-496.

27. Clergue F, Mantembault C, Despierres 0 , et al. Respiratory effects of intrathecal morphine after upper abdominal surgery. Anesthesiology 1984; 61: 677-685.

28. Benhamou D, Samii K, Noviant Y. Effects of analgesia on respiratory muscle function after upper abdominal surgery. Acta Anaesthesiol Scand 1983; 27: 22-25.

29. Ellström M, Fagevik Olsén M, Olsson J-E, et al. Pain and pulmonary function following laparoscopic and abdominal hysterectomy: a randomized study. Acta Obstet Gynecol Scand 1988; 77: 923-928.

30. Fagevik Olsén M, Josefson $K$, Lönroth $H$. Chest physiotherapy does not improve the outcome in laparoscopic fundoplication and vertical-banded gastroplasty. Surg Endosc 1999; 13: 260-263.

31. Olbers T, Fagevik Olsén M, Maleckas A, Lönroth H. Randomized clinical trial of laparoscopic Roux-en-Y gastric by-pass versus laparoscopic vertical banded gastroplasty for obesity. Br J Surg 2005; (in press).

32. Fagevik Olsén M, Wennberg $E$, Johnsson $E$, et al. Prevention of pulmonary complications after thoracoabdominal resections by two different breathing techniques. Br J Surg 2002; 89: 1228-1234.

33. Quanjer Ph H, Tammeling GJ, Cotes JE, et al. Lung volumes and forced ventilatory flows. Eur Respir J 1993; 6: Suppl 16, 5-40.

34. Giroux, JM. Lewis S, Holland LG, et al. Postoperative chest physiotherapy for abdominal hysterectomy patients. Physiotherapy Canada 1987; 39: 89-93.

35. Fagevik Olsén M. Chest physiotherapy in open and laparoscopic abdominal surgery. Phys Ther Rev 2000; 5: 125-130. 